\title{
Detection of egg stains based on local texture feature clustering
}

\author{
Qinghua Yang, Mimi Jia, Yi Xun, Guanjun Bao* \\ (Key Laboratory of E\&M, Ministry of Education \& Zhejiang Province, Zhejiang University of Technology, Hangzhou 310032, China)
}

\begin{abstract}
The quality of egg is mainly influenced by the dirt adhering to its shell. Even with good farm-management practices and careful handling, a small percentage of dirty eggs will be produced. The purpose of this research was to detect the egg stains by using image processing technique. Compared to the color values, the local texture was found to be much more adept at accurately segmenting of the complex and miscellaneous dirt stains on the egg shell. Firstly, the global threshold of the image was obtained by two-peak method. The irrelevant background was removed by using the global threshold and the interested region was acquired. The local texture information extracted from the interested region was taken as the input of fuzzy C-means clustering for segmentation of the dirt stains. According to the principle of projection, the area of dirt stains on the curved egg surface was accurately calculated. The validation experimental results showed that the proposed method for classifying eggs in terms of stain has the specificity of $91.4 \%$ for white eggs and $89.5 \%$ for brown eggs.
\end{abstract}

Keywords: eggs, eggshell dirt stains, computer vision, local texture feature, FCM, egg classifying

DOI: $10.25165 /$ j.ijabe.20181101.2592

Citation: Yang Q H, Jia M M, Xun Y, Bao G J. Detection of egg stains based on local texture feature clustering. Int J Agric \& Biol Eng, 2018; 11(1): 199-205.

\section{Introduction}

So far, the collection and packaging of fresh eggs have been automated, and the related researches on detecting egg weight, size and infertile hatching eggs have been realized ${ }^{[1-3]}$. However, there is still a need for further study of egg defects detection. The three main quality defects include cracks on eggshell, internal blood spots and external dirt stains. With regard to the detection of cracked eggs, great quantities of studies have found that the acoustic characteristics can be used to identify damaged eggs effectively ${ }^{[4,5]}$. For the detection of internal blood spot, the spectral method was proved valid by previous studies ${ }^{[6]}$. And the related theories have been applied to current commercial systems ${ }^{[7]}$. The application of computer vision techniques in dirt stains detection showed well effect in practice.

During the process of production, storage and transportation, eggs may be polluted by blood from a prolapsed cloaca, faecal, water stains, grease, oil stains, etc ${ }^{[8]}$. Dirt stains may exist in different types of pathogenic bacteria, such as Salmonella, Escherichia coli and Staphylococcus, which may cause serious foodborne illness ${ }^{[9,10]}$. Moreover, cross-contamination may occur in the process of transportation and storage of eggs. The eggshell cleanliness is an important index of egg grading.

With the development of image processing technology and computer hardware, machine vision has shown great potential in detecting dirty eggs. Up to now, a number of studies have been conducted to detect egg stains based on machine vision. In 1996,

Received date: 2016-05-22 Accepted date: 2016-09-07

Biographies: Qinghua Yang, $\mathrm{PhD}$, Professor, research interests: robot, Email: zjutme@163.com, king@zjut.edu.cn; Mimi Jia, Master candidate, research interests: machine vision, Email: jtom_email@163.com; Yi Xun, PhD, Lecturer, research interests: machine vision, Email: xunyi@zjut.edu.cn.

*Corresponding author: Guanjun Bao, $\mathrm{PhD}$, Professor, research interests: robot. Zhejiang University of Technology, China. Tel: +86-571-88320819, Email: gjbao@zjut.edu.cn.
Patel et al. ${ }^{[11]}$ proposed the neural network model trained by gray histogram for egg surface defects detection. Although such model for dirt stain detection had an average accuracy of $80.0 \%$, the detection of single egg cost more than $1000 \mathrm{~ms}$. Then Patel et al. ${ }^{[12]}$ improved the neural network model to be trained by color histogram in 1998, which increased the average accuracy for dirt stain detection to $85 \%$. In 2000 , Garcia-Alegre et al. ${ }^{[13]}$ found that subtracting background estimation obtained by average filtering from $[(\mathrm{R}-\mathrm{B}) /(\mathrm{R}+\mathrm{B})]$ the normalized color differential image can help to enhance dirt stains and cracks on the eggshell and effectively increased the detection speed. The same year, Ribeiro ${ }^{[14]}$ proposed GA classification algorithm based on sample learning, which can identify the egg stains more accurately. Mertens et al. ${ }^{[15]}$ designed an off-line computer vision detection system to identify different types of dirt on brown eggshell including dark (feces), white (uric acid), blood, and yolk stains, with the accuracy recognition rate of 99\%. Recently, new researches have shown the possibility to distinguish organic stains from natural stains. In 2012, Lunadei et al. ${ }^{[16]}$ opportunely combined monochromatic images to detect cracks and egg stains with high classification rate of $98 \%$ and low processing time $(0.05 \mathrm{~s})$. To avoid the influence of uneven brightness caused by curved egg surface, Arivazhagan et al. ${ }^{[17]}$ proposed to extract $\mathrm{H}$ component from HSV color space and then use the global threshold segmentation method. However it is practically difficult to identify the black stains adhering to the egg surface.

Some of above-mentioned studies were only applicable to specific shell color or specific type of dirt stains. Owing to the problems mentioned above, this study adopted local texture information instead of colors information to accurately segment the dirt stains on the egg shell. The research aimed to design and evaluate an egg detection system for distinguishing dirty and clean eggs, eliminating the disturbance of eggshell color and dirt stains on the detection precision and improving the average accuracy of dirt stains detection. 


\section{Materials and methods}

\subsection{Sample preparation}

In this research, a total number of 300 chicken eggs with different shell colors (white and brown) were selected from a farmers market in Hangzhou, China. Egg samples with normal surface, and four common external dirt stains (i.e. bloody speckle, fecal matter, urine, chicken feed, dripping liquid from the leakers) were collected. The dirty eggs were inspected and classified by skilled egg graders. Among these egg samples, there were 154 clean eggs and 146 defected eggs with complex types of dirt stains. The detailed artificial classification results of 300 egg samples are shown in Table 1.

Table 1 Artificial classification results of 300 egg samples

\begin{tabular}{clccc}
\hline & & \multicolumn{2}{c}{ Eggshell color } & \multirow{2}{*}{ Total } \\
\cline { 3 - 4 } & & White & Brown & \\
\hline \multirow{3}{*}{$\begin{array}{c}\text { Sample } \\
\text { types }\end{array}$} & Bloody Speckle & 15 & 7 & 22 \\
& Fecal matter and urine & 21 & 25 & 46 \\
& Chicken feed & 19 & 24 & 43 \\
& Dripping Liquid & 15 & 20 & 35 \\
& Clean & 70 & 84 & 154 \\
\hline & Total & 140 & 160 & 300 \\
\hline
\end{tabular}

Figure 1 shows the samples of dirty eggs with common dirt stains. The blood stains are more dispersed and deep red in appearance, its boundary adjacent to the clean region is fuzzy. Fecal matter, urine and chicken feed are the most common stains on the egg surface, which have diverse colors, but they are more concentrated that relative to the blood stains. Dripping liquid stains refers to yolk and albumen adhering to the eggshell. Such stains, albumen in particular, have a quite similar color to eggshell due to its transparent characteristic.

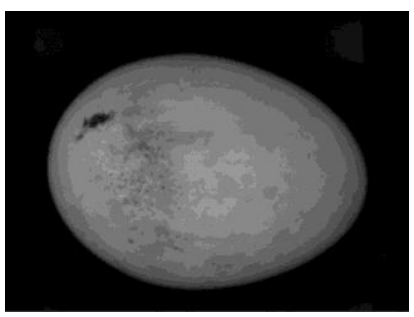

a. Bloody speckle

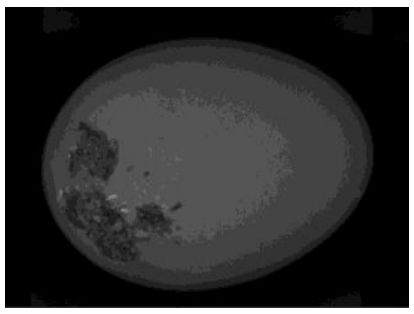

c. Chicken feed

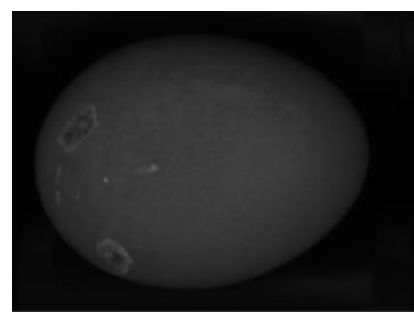

b. Fecal matter and urine

d. Dripping liquid

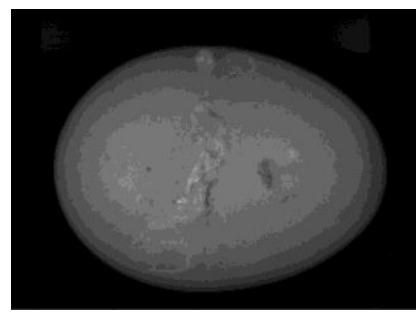

Figure 1 Different types of dirt stains on the eggshell

\subsection{Computer vision system}

The egg detection system based on computer vision was developed, as shown in Figure 2. It was mainly composed of mechanical transmission, illumination chamber, industrial digital camera, fluorescent tubes and computer. Three rows of eggs were placed on the dual tapered rollers which moved forward on the chain. The length of each roller was $72 \mathrm{~mm}$ and the interval between them was $54 \mathrm{~mm}$. Once the egg entered the illumination chamber, the dual tapered rollers contacted with the friction tape and rotated clockwise. The eggs on the rollers are driven to roll around the axis $A-A$ ' counter clockwise as shown in Figure 2. The camera was triggered to acquire images of eggs, making that three sides of each egg would be captured in the illumination box. Almost full details about the egg surface can be obtained from three sides of egg. So $3 \times 3$ eggs should be captured in one single image at the same time.

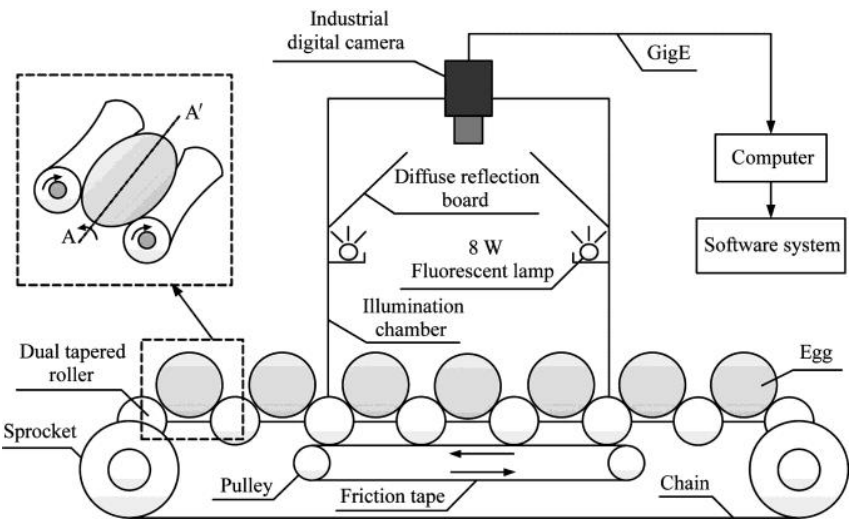

Figure 2 Egg detection system based on computer vision

The illumination chamber was designed with the size of $350 \mathrm{~mm} \times 350 \mathrm{~mm} \times 350 \mathrm{~mm}$, larger than the required size in order to adapt to the length of fluorescent tube (Field of view was still $216 \mathrm{~mm} \times 162 \mathrm{~mm}$ ). Four fluorescent tubes (Philips, $8 \mathrm{~W}, 220 \mathrm{~V}$ ) were distributed on all four inner surface of the illumination chamber, and diffuse reflection boards were mounted ahead of the fluorescent tubes to ensure the uniformity of illumination ${ }^{[18]}$. The Basler acA2500-14gm monochrome industrial digital camera was selected as image acquisition device, which has a maximum resolution of $2592 \times 1944$ pixels. Fixed size segmentation was used to get each individual egg image with the size of $864 \times 648$ pixels, as shown in Figure 3.

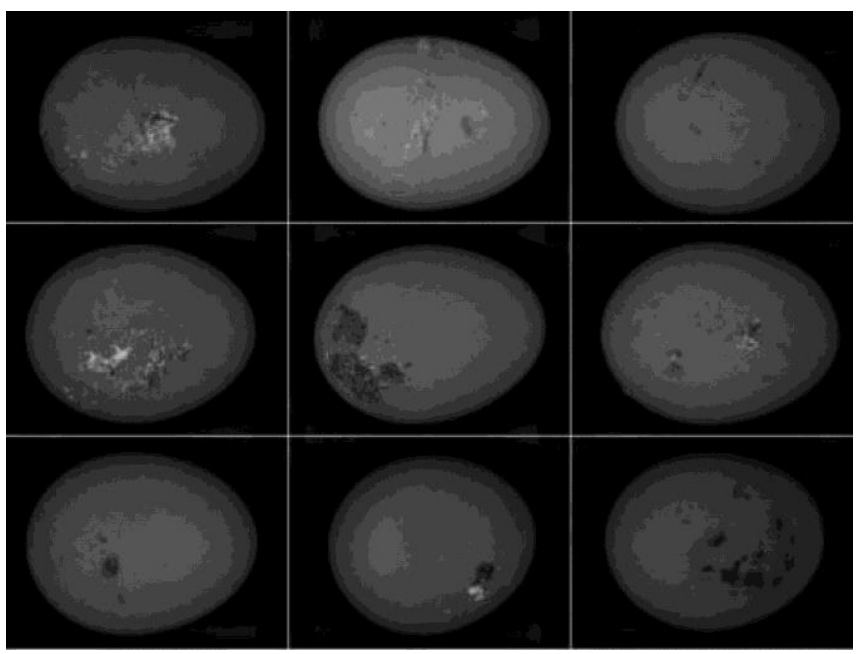

Figure 3 Fixed size segmentation

\section{Image processing}

\subsection{Egg image pre-processing}

The original image was firstly processed with the circular averaging filter (with radius 3) to filter out noise on the image. Additionally, in order to speed up the image processing and eliminate the influence of background on the post processing, the background should be removed. The background region has much lower gray value than the egg region, so gray histogram of the egg image has marked peaks and valley. Hence, the valley signal of the gray histogram was regarded as global threshold to 
differentiate target region and background region.

Black dirt stains on the egg shell have similar gray values with background. As Figure $4 \mathrm{~b}$ shows, part pixels of the egg image were mistakenly segmented. To address this issue, the maximum external contour of the binary image was traced to separate the target region from background. As shown in Figure $4 \mathrm{c}$, the internal region of maximum external contour was filled with white pixels to eliminate the black holes ${ }^{[15]}$, while the external region was filled with black pixels.

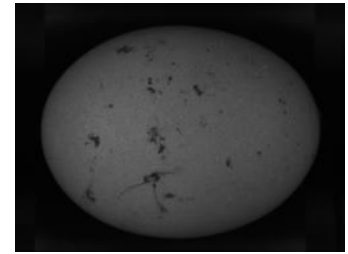

a. Original image after filtering

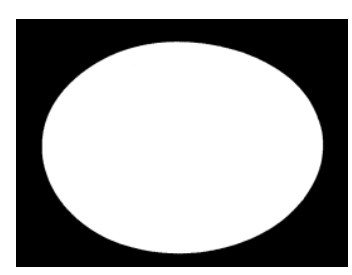

c. Image after filling holes

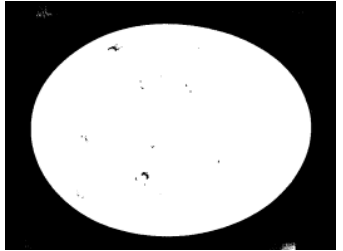

b. Binary image

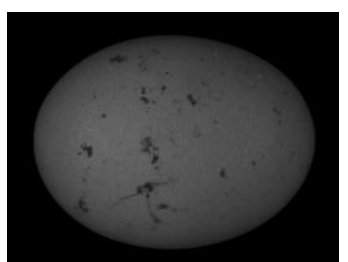

d. Image after removing background
Figure 4 Pre-processing steps for egg image

\subsection{Dirt stains segmentation}

\subsubsection{Texture features extraction}

The surface of egg is curved, which makes the brightness of target region uneven (Figure 5a). And more importantly, the dirt stains on the egg shell are complex and miscellaneous, which include feces, dirt and blood from a prolapsed cloaca or yolk of damaged egg. Therefore, it is not reliable to detect a variety of dirt stain defects by traditional threshold segmentation algorithm. In addition to the gray-scale information, the texture features are also widely used in scientific research. It was obvious that the surface of clean egg is smooth, whereas the dirt stains region is irregular rough.

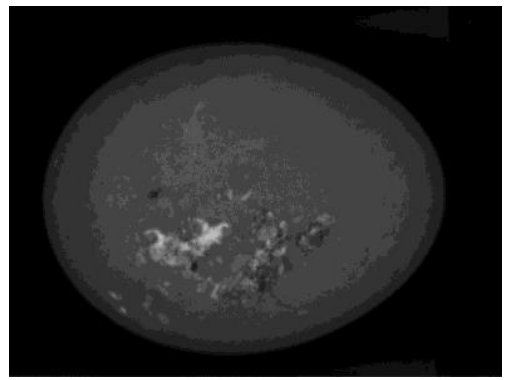

a. Original image

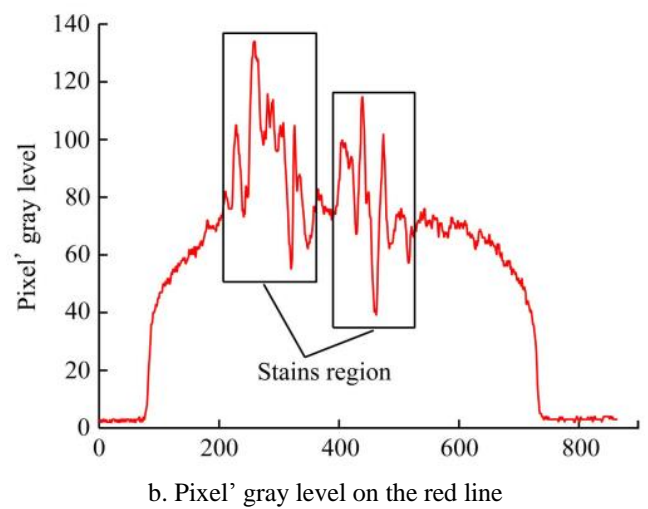

Figure 5 Analysis of stained egg's gray level
The statistical moment of the gray histogram of image is the simplest way to describe the texture ${ }^{[19]}$. Texture descriptors based on gray histogram include: (1) Mean value $m$ : measure average brightness; (2) Standard deviations $\sigma$ : measure average contrast; (3) Smoothness $R$ : measure relative smoothness; (4) Consistency $U$ : measure regional gray level consistency; (5) Entropy $e$ : measure regional gray level randomness.

$$
\begin{gathered}
m=\sum_{i=0}^{L-1} z_{i} p\left(z_{i}\right) \\
\sigma=\sqrt{\mu_{2}(z)}=\sqrt{\sum_{i=0}^{L-1}\left(z_{i}-m\right)^{2} p\left(z_{i}\right)} \\
R=1-1 /\left(1+\sigma^{2}\right) \\
U=\sum_{i=0}^{L-1} p^{2}\left(z_{i}\right) \\
e=-\sum_{i=0}^{L-1} p\left(z_{i}\right) \log _{2} p\left(z_{i}\right)
\end{gathered}
$$

where, $z_{\mathrm{i}}$ denotes the random variables of image gray level, $p\left(z_{\mathrm{i}}\right)$, stands for corresponding gray histogram, $i=0,1,2, \ldots, L-1, L$ is the number of distinguished gray levels.

Equation (4) obtained the image gray level consistency $U$, the higher the consistency, the greater the $U$. In order to obtain image gray inconsistency, the Equation (4) has been rewritten to highlight the dirt stains region:

$$
U^{\prime}=\sum_{i=0}^{L-1}\left(1 / p\left(z_{i}\right)\right)^{2}
$$

The overall texture of the egg surface is scarcely influenced by small stains (Figure 5b). Considering the uncertainty of the stain size, the local texture features were used to analyze the egg image. The texture features of each pixel can be obtained by calculating the statistical moment of the gray histogram of its neighboring pixels. To improve calculation speed, the image was divided into blocks, and the texture features of each block were calculated ${ }^{[20]}$. Then, the local texture features of each pixel were obtained by bi-linear interpolation. Such method can both improve the calculation speed and ensure the accuracy of image segmentation. The results were normalized as shown in Figure 6 (block size $=$ $4 \times 4)$.

The extracted features are of high dimensions, so the dimensionality reduction is necessary before classification. The mean value of dirt stains region may be higher or lower than the clean region. Therefore, the local mean texture cannot distinguish between dirt stains region and clean region. In Figure $6 \mathrm{c}$, the smoothness texture of clean region and most dirt stains region are both close to 0 . If the smoothness texture was chosen as clustering feature, most of the dirt stains would be detected incorrectly as clean region. Figure $6 e$ shows that the local entropy of partial clean region is close to dirt stains region. Similarly, supposing that the local entropy was chosen as clustering feature, most of the clean region would be detected incorrectly as the dirt stains. Comparing Figures $6 \mathrm{~b}$ and $6 \mathrm{~d}$ (the standard deviation texture map and inconsistency texture map), there is a notable difference between the clean region and the dirt stains region of egg image. So the feature vector $x_{i}=\left[\sigma, U^{\prime}\right], i=1,2, \ldots, m \times n$ was adopted, where $m$ and $n$ respectively represent the height and width of the image, $\sigma$ and $U^{\prime}$ respectively represent normalized standard deviation feature and gray inconsistency feature. 


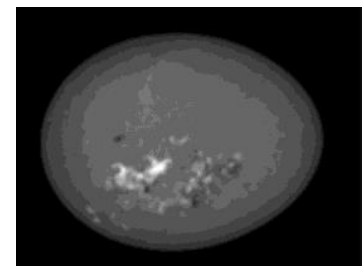

a. Mean texture map

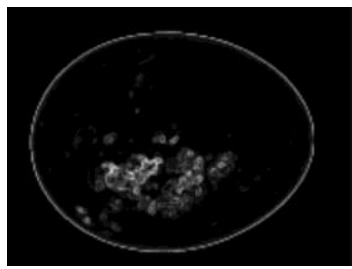

b. Standard deviation texture map

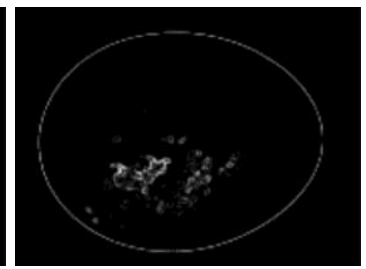

c. Smoothness texture map

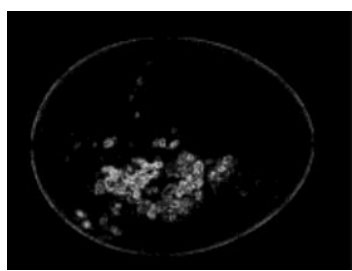

d. Inconsistency texture map

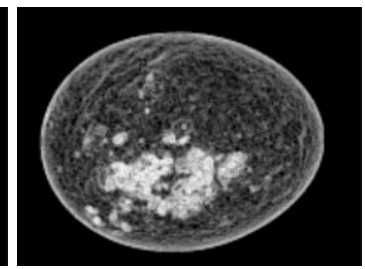

e. Entropy texture map

Figure 6 Local texture of egg image

\subsubsection{Clustering analysis and dirt stains segmentation}

As mentioned above, standard deviation $\sigma$ and inconsistency texture feature $U^{\prime}$ were chosen as the input features of FCM (Fuzzy C-Means Clustering). The core idea of FCM is: dividing $N$ vectors $x_{\mathrm{i}}(i=1,2, \ldots, N)$ into $C$ classes to minimize the objective function. The objective function is:

$$
J_{m}=\sum_{i=1}^{N} \sum_{j=1}^{c} u_{i j}^{m}\left\|x_{i}-c_{j}\right\|^{2}(1 \leq m<\infty)
$$

where, $m=2, u_{\mathrm{ij}}$ is the membership degree of the $i^{\text {th }}$ sample $x_{\mathrm{i}}$ belonging to the class $j ; c_{\mathrm{j}}$ is a clustering center of class $j,\|*\|$ represents the distance between the sample data and the cluster center. In this research, we chose Euclidean distance as a measure of similarity. FCM was used to cluster the pixels of the target region, and the points in the feature space were divided into 2 groups, which respectively represent the clean region and the dirt stains region, as shown in Figure 7.

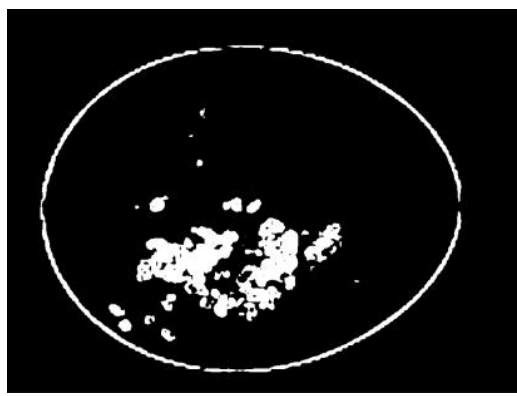

Figure 7 Segmentation result by clustering

After the image segmentation based on FCM, the dirt stains adhering to the egg shell would be segmented accurately. Owing to the dramatic gray transition, the edge of egg target was divided into dirt stains incorrectly. It is necessary to remove the edge region from the segmentation image. Considering the fact that the false segmented region always exists on the edge, a slightly smaller mask can be used to remove the wrongly segmented region. The required mask was obtained by Erosion operation on the binary

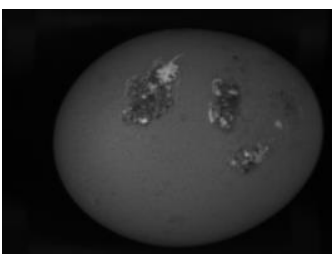

a. Original image

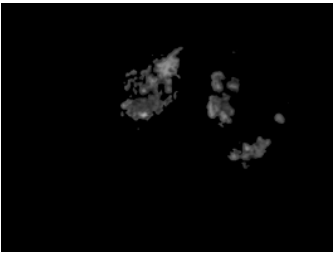

e. Block size $=6 \times 6$

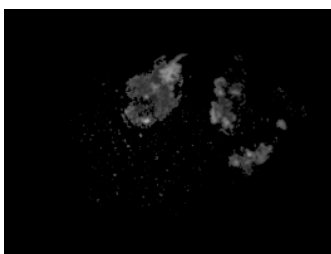

b. Block size $=3 \times 3$

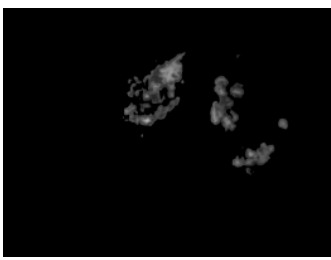

f. Block size $=7 \times 7$

image which was acquired on the image pre-processing step. Furthermore, holes in the dirt stains region are likely to be wrongly segmented. By means of morphology, the holes in the connected area can be filled. The process is shown in Figure 8.

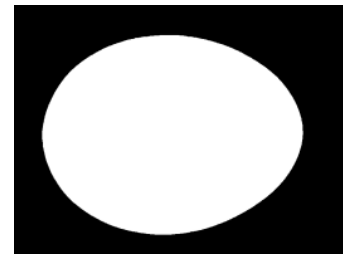

a. Required mask

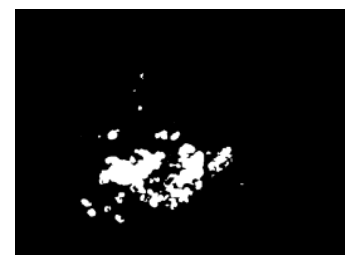

c. Segmentation image after filling holes

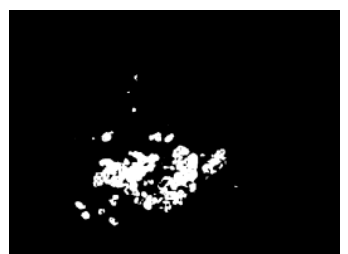

b. Segmentation image after removing edge

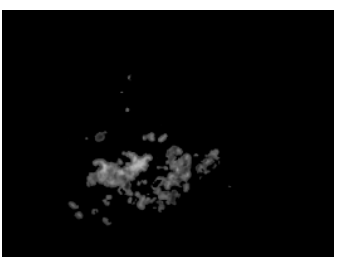

d. Result of dirt stains segmentation

Figure 8 Removal process of false segmentation region

\subsubsection{Selection of algorithm parameters}

The choice of the block size is the critical part of the proposed algorithm to segment egg dirt stains based on local texture feature clustering. The block size used in the description of the algorithm was $4 \times 4$. Then the effect of block size has been demonstrated on the segmentation, as shown in Figure 9.

When the block size is too small, lots of clean pixels are misclassified as dirt stains. With the block size increasing, the clean region outside the dirt stain edge is wrongly segmented as dirt stains. The block size of $5 \times 5$ after a series of test has been chosen.

\subsection{Algorithm segmentation effect}

In order to verify the general applicability of the proposed algorithm to various dirt stains, egg images with different shell colors and different types of dirt stains were segmented by the proposed method, as shown in Figure 10.

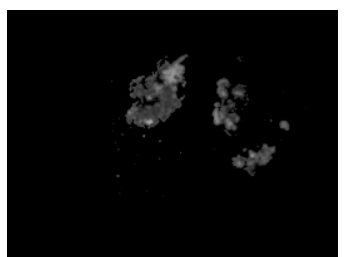

c. Block size $=4 \times 4$

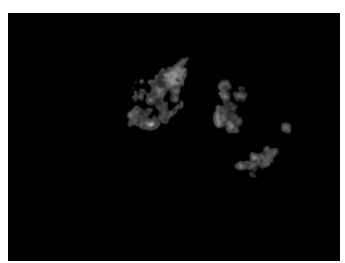

g. Block size $=8 \times 8$

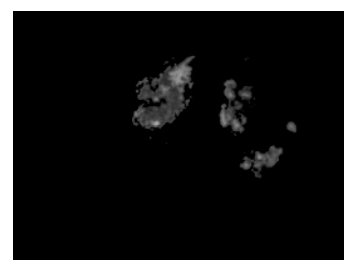

d. Block size $=5 \times 5$

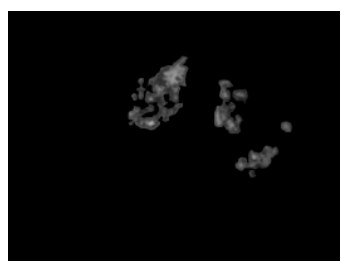

h. Block size $=9 \times 9$

Figure 9 Comparison of segmentation results with different block sizes 

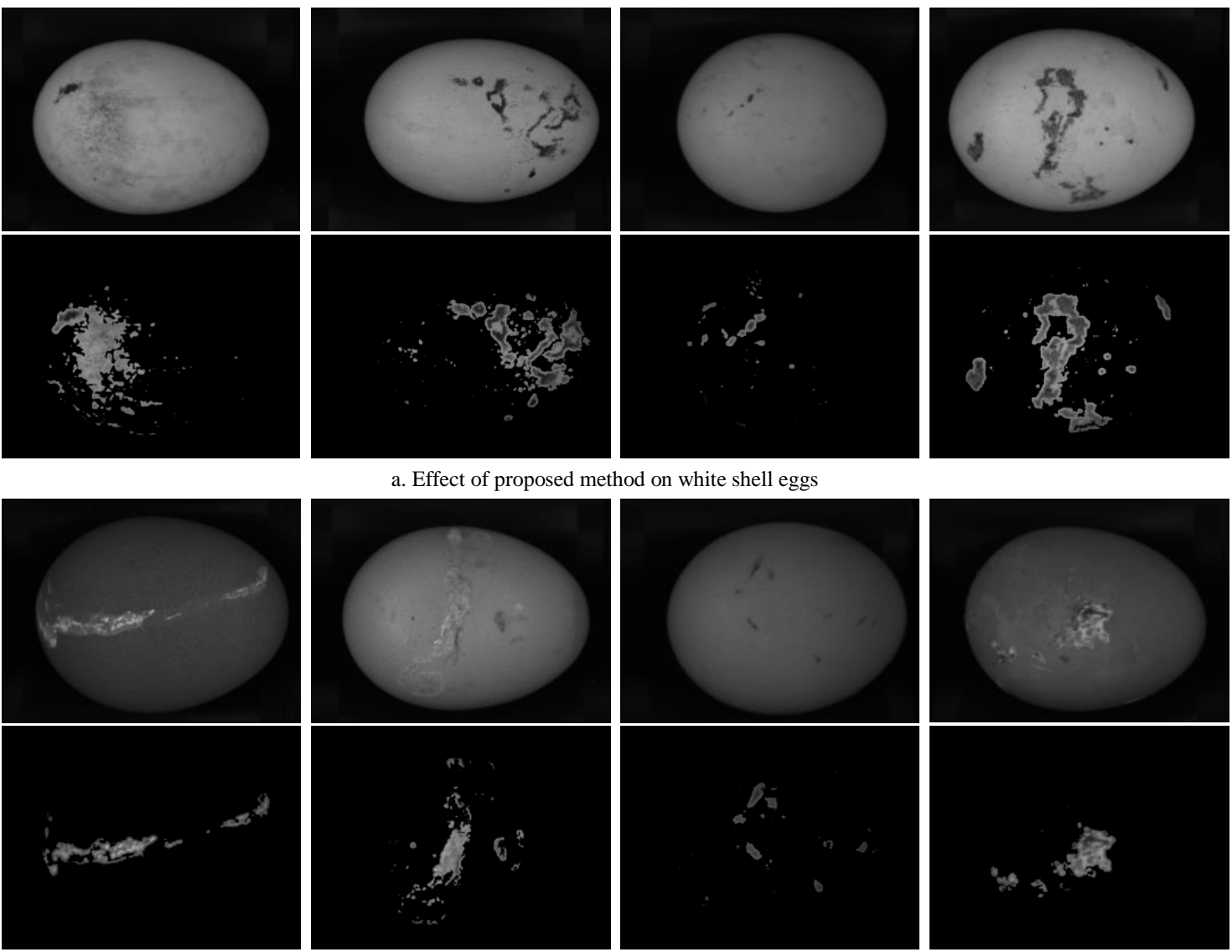

b. Effect of proposed method on brown shell eggs

Figure 10 Segmentation effect of different eggshell color with various dirt stains

Experimental results showed that the proposed method can segment various types of egg dirt stains with different shapes or intensity.

\section{Classifying method}

\subsection{Calculation of dirt stains region area}

The severity of egg shell dirt is different in eggs, for this case the severity detection of defects is necessary. By using the proposed method, the dirt stains on the egg shell can be accurately segmented. Figure 9d demonstrates the fact that some noises were generated using the proposed method, which are much smaller than the real dirt stains. Hence, small connected regions were ignored by calculation of the area of each dirt stains region. Experimental results indicated that the region with an area of less than 20 pixels can be considered as noise.

At present, the surface of egg was treated as a plane, which would cause large deviation in detection results. Apparently the dirt stain in the center of the egg surface and the dirt stain of equal size on the edge of the egg will produce different results. By calculating the dirt stains region area of the segmented egg image, we can quantitatively describe the severity of egg shell dirt. Therefore, it is necessary to calculate the actual area of the dirt stain according to the image information.

As shown in Figure 11, it is assumed that the surface of an egg is performed by rotation of the outline $A C B$ of projected area in line of longitudinal axis $A B^{[1]}$. The captured image was the projection of egg surface in $X O Y$ plane, each pixel of which was regarded as a square with an area of $1^{[21]}$. According to the projection angle of each pixel in the egg region, we can calculate the actual area of dirt stain. The coordinates of the actual point on the egg surface can be calculated. The $\Delta O^{\prime} S S^{\prime}$ in Figure 11 is an right triangle with the side length: $O^{\prime} S^{\prime}=\left(L_{1}+L_{2}\right) / 2, O^{\prime} S=\left(L_{1}-L_{2}\right) / 2$. According to the Pythagorean theorem, it can be obtained: $S S^{\prime}=L_{1} L_{2}{ }^{1 / 2}$.

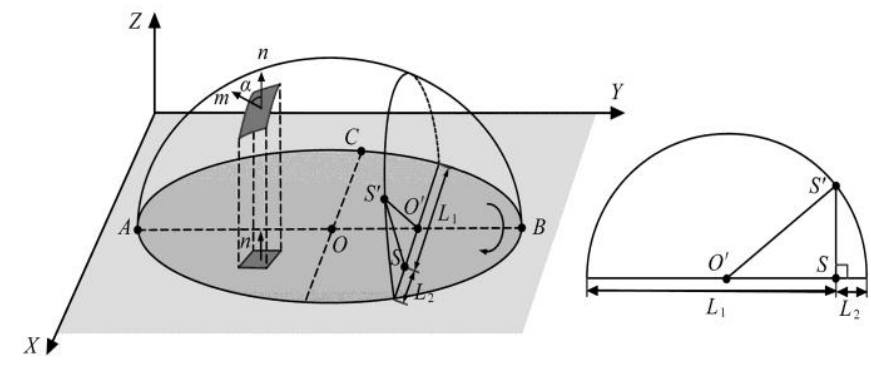

Figure 11 Calculation of actual area for egg surface

Assuming that any point $(x, y, z)$ on the egg surface satisfies:

$$
F(x, y, z)=z-f(x, y)=0
$$

The normal vector $\boldsymbol{m}$ of the point $\left(x_{0}, y_{0}, z_{0}\right)$ on the egg surface is obtained as follows:

$$
\left.\boldsymbol{m}\right|_{\left(x_{0}, y_{0}, z_{0}\right)}=\left(\frac{\partial F}{\partial x}, \frac{\partial F}{\partial y}, \frac{\partial F}{\partial z}\right)=\left(-\frac{\partial f}{\partial x},-\frac{\partial f}{\partial y}, 1\right)
$$

The normal vector of the plane $X O Y$ is: $\boldsymbol{n}=(0,0,1)$. The cosine of the projection angle of the egg surface is:

$$
\cos \alpha=\boldsymbol{m} \cdot \boldsymbol{n} /(|\boldsymbol{m}| \times|\boldsymbol{n}|)=1 / \sqrt{\left(\frac{\partial f}{\partial x}\right)^{2}+\left(\frac{\partial f}{\partial y}\right)^{2}+1}
$$

Although the function expression of $z=f(x, y)$ was unknown, the coordinates of each point on the surface had been calculated, therefore we can use finite differences to calculate the partial derivatives:

$$
\frac{\partial f}{\partial x}=f(x+1, y)-f(x, y)
$$




$$
\frac{\partial f}{\partial y}=f(x, y+1)-f(x, y)
$$

The actual area of each pixel in the egg region can be calculated according to the Equation: $\Delta S=1 / \cos \alpha$. The actual area of each connected region can be obtained by accumulating the actual area of each pixel in the connected area.

\subsection{Establishment of classifying method}

In order to eliminate the influence of egg size on the experimental results, this study regards the ratio of the dirt stains region area $S_{\mathrm{z}}$ and egg region area $S$ as identification parameter to establish the classifying standard ${ }^{[22]}$.

$$
K=\frac{S_{z}}{S}
$$

So-called dirty egg is a shell that is unbroken and has dirt or foreign material adhering to its shell, which has prominent stains, or moderate stains covering more than $1 / 32$ of the shell surface if localized, or 1/16 of the shell surface if scattered (USDA, 2000) ${ }^{[23]}$. To ensure safety and quality of eggs, the threshold was fixed on 0.03. In this research, three faces of each single egg were captured. When any one of them satisfied $K \geq 0.03$, we can distinguish the egg as dirty egg.

\subsection{Classifying experiment}

The main work of this research was to develop a new segmentation algorithm for the detection of eggshell dirt stains. The emphasis here should be on eliminating the influence of various eggshell colors and complicated shapes, distribution, intensity of external stains.

Totally 300 eggs were collected as test set to verify the effectiveness of the classifying method, which contained 154 clean eggs and 146 dirty eggs by manual testing. The types of dirt stains included bloody speckle, fecal matter, urine, chicken feed and dripping liquid from the leakers. Table 2 shows the detection results for white eggs and brown eggs using the proposed method. Accuracy is the number of correctly predicted eggs divided by the total number of egg samples. Sensitivity is the measure of the ability of the proposed method to recognize the positive cases. Specificity is the measure of the ability of the proposed method to recognize the negative cases. The results showed that the classifying method had an adequate performance for eggs with different shell colors. Comparing with some previous works focused on the detection of eggs with specific $\operatorname{color}^{[11,12]}$, the proposed method has behaved strong flexibility.

Table 2 Test results of the proposed classification method

\begin{tabular}{ccccccc}
\hline \multirow{2}{*}{ Class } & \multicolumn{2}{c}{ Predicted } & & & \\
\cline { 3 - 5 } & & $\begin{array}{c}\text { Clean } \\
\text { eggs }\end{array}$ & $\begin{array}{c}\text { Dirty } \\
\text { eggs }\end{array}$ & $\begin{array}{c}1 \% \\
\text { Wharacy }\end{array}$ & $\begin{array}{c}\text { Sensitivity } \\
1 \%\end{array}$ & $\begin{array}{c}\text { Specificity } \\
1 \%\end{array}$ \\
\hline \multirow{2}{*}{ White } & Clean eggs & 68 & 2 & 94.3 & 97.1 & 91.4 \\
& Dirty eggs & 6 & 64 & & & \\
\hline Brown & Clean eggs & 76 & 8 & 90.0 & 90.5 & 89.5 \\
\hline
\end{tabular}

The proposed method misclassified 10 clean eggs in dirty class and 14 dirty eggs in clean class. After analysis, 10 clean eggs misclassified as dirty eggs were mainly attached by wrinkles or spots. However, such wrinkles or spots are different in texture with clean regions. The proposed segmentation algorithm may falsely segment them as dirt stains. Fourteen dirty eggs, mostly eggs with dripping liquid, were misclassified as clean eggs. Besides, the two ends of egg were hard to be captured. Dirt stains adhering to the ends of egg may be ignored by visual detection.

The detection results of common types of eggshell dirt stains were shown in Table 3. In this case, the proposed method was suitable to detect such types of eggshell stains, apart from the dirty eggs with dripping liquid (near $85.7 \%$ of correctly classified) and bloody speckle (near 90.9\%). Due to dispersed distribution and small size of scale, the blood speckles may be removed as noise in process of spot area calculation. The cases of misclassification of eggs with dripping liquid were different: what happened here is that the color of these stains was quite similar to eggshell, and sometimes there were no obvious texture differences between them. Garcia-Alegre et al. ${ }^{[13]}$ reported an accuracy of $92 \%$ for dirty eggs and $82 \%$ for clean ones. Dehrouyeh et al. ${ }^{[8]}$ obtained an accuracy of $85.66 \%$ for dirty eggs. However, such studies can not be applied on various types of dirt stains and were incapable of detecting dark stains effectively.

Table 3 Detection results of common types of eggshell dirt stains

\begin{tabular}{lccc}
\hline \multicolumn{1}{c}{ Sample types } & No. of samples & $\begin{array}{c}\text { No. of } \\
\text { misclassified }\end{array}$ & $\begin{array}{c}\text { Classification } \\
\text { accuracy/\% }\end{array}$ \\
\hline Bloody speckle & 22 & 2 & 90.9 \\
Fecal matter and urine & 46 & 4 & 91.3 \\
Chicken feed & 43 & 3 & 93.0 \\
Dripping liquid & 35 & 5 & 85.7 \\
Clean & 154 & 10 & 93.5 \\
\hline
\end{tabular}

In the actual production activities, the most important indicator is specificity. It would rather distinguish the clean egg to be dirty egg than identify the dirty egg incorrectly as clean one and finally appear in the products. The proposed method to classify eggs has the specificity of $91.4 \%$ for white eggs and $89.5 \%$ for brown eggs. The results showed that the classifying method had an adequate performance.

\section{Conclusions}

(1) In view of the fact that it is difficult to adapt to all kinds of dirt stains using traditional threshold segmentation, the new dirt stain segmentation method based on local texture was proposed. Fuzzy C-means clustering was performed with the input features of local standard deviation and local inconsistency texture. The results showed that the method had good result on the segmentation of various kinds of dirt stains.

(2) According to the principle of projection, the area of dirt stains on the curved egg surface was accurately calculated. When the stains region area is greater than 0.03 of each side face of egg, the egg is determined as dirty egg. Experimental results indicated that the proposed method to classify eggs had the specificity of $91.4 \%$ for white eggs and $89.5 \%$ for brown eggs.

Although the accuracy of proposed dirty egg classification method has not the requirements of egg automatic online detection, the work carried out in this paper is meaningful. Method to detect external defects based on local texture can be used not only for detecting poultry eggs, but also for other objects with smooth surface. In addition, the area projection algorithm can be used to calculate the actual surface area of regular curved objects, and effectively improve the detection accuracy of surface defects. Further studies may focus on increasing the classification accuracy by using appropriate features and machine learning algorithm.

\section{Acknowledgments}

The authors gratefully acknowledge the financial support of the National Science \& Technology Pillar Program (2015BAD19B05). 


\section{[References]}

[1] Soltani M, Omid M, Alimardani R. Egg volume prediction using machine vision technique based on pappus theorem and artificial neural network. Journal of Food Science and Technology, 2015; 52(5): 3065-3071.

[2] Zhang W, Wu X, Qiu Z, He Y. A novel method for measuring the volume and surface area of egg. Journal of Food Engineering, 2015; 170: 160-169.

[3] Zhu Z H, Liu T, Xie D J, Wang Q H, Ma M H. Nondestructive detection of infertile hatching eggs based on spectral and imaging information. Int J Agric \& Biol Eng, 2015; 8(4): 69-76.

[4] Ketelaere B D, Coucke P, Baerdemaeker J D. Eggshell crack detection based on acoustic resonance frequency analysis. Journal of Agricultural Engineering Research, 2000; 76(2): 157-163.

[5] Cheng J, Xie L, Ying Y. Eggshell crack detection based on the time-domain acoustic signal of rolling eggs on a step-plate. Journal of Food Engineering, 2015; 153(1): 53-62.

[6] Chen M, Zhang L, Xu H. On-line detection of blood spot introduced into brown-shell eggs using visible absorbance spectroscopy. Biosystems Engineering, 2015; 131: 95-101.

[7] Detector: Abnormal Egg Detector. Available: https://www.nabel.co.jp/ english/product/abd.html. Accessed on [2016/5/10]. (in Chinese)

[8] Dehrouyeh M H, Omid M, Ahmadi H, Mohtasebi S S, Jamzad M. Grading and quality inspection of defected eggs using machine vision. International Journal of Advanced Science \& Technology, 2010; 16: 23-30.

[9] Mor-Mur M, Yuste J. Emerging bacterial pathogens in meat and poultry: An overview. Food and Bioprocess Technology, 2010; 3(1): 24.

[10] Wesley I V, Muraoka W T. Time of entry of salmonella, and campylobacter, into the Turkey Brooder House. Food and Bioprocess Technology, 2011; 4(4): 616-623.

[11] Patel VC, McClendon R W, Goodrum J W. Detection of blood spots and dirt stains in eggs using computer vision and neural networks. Applied Engineering in agriculture, 1996; 12(2): 253-258.

[12] Patel V C, Mcclendon R W, Goodrum J W. Color computer vision and artificial neural networks for the detection of defects in poultry eggs. Artificial Intelligence Review, 1998; 12(1): 163-176.

[13] Garcia-Alegre M C, Ribeiro A, Guinea D, Cristobal G. Eggshell defects detection based on color processing. Proceedings of SPIE - The International Society for Optical Engineering, 2007; 3966: 280-287.

[14] Ribeiro A, García-Alegre M C, Guinea D, Cristobal G. Automatic rules generation by GA for eggshell defect classification. Networks, 2000; 4: 5 .

[15] Mertens K, Ketelaere B D, Kamers B, Bamelis F R, Kemps B J, Verhoelst E M, et al. Dirt detection on brown eggs by means of color computer vision. Poultry Science, 2005; 84(10): 1653-1659.

[16] Lunadei L, Ruiz-Garcia L, Bodria L, Guidetti R. Automatic identification of defects on eggshell through a multispectral vision system. Food and Bioprocess Technology, 2012; 5(8): 3042-3050.

[17] Arivazhagan S, Shebiah R N, Sudharsan H, Kannan R R, Ramesh R. External and internal defect detection of egg using machine vision. Journal of Emerging Trends in Computing and Information Sciences, 2013; 4(3): 257-262.

[18] Cen Y K. Research on quality inspection of eggs based on machine vision. Master dissertation. Hangzhou: Zhejiang University, 2006. (in Chinese)

[19] Ma L, Fan Y L. Texture image analysis. Beijing: Science Press, 2009. p231. (in Chinese)

[20] Wang W F, Ma L, Yang L. Liver contour extraction using modified snake with morphological multiscale gradients. 2008 International Conference on Computer Science and Software Engineering, 2008; 6: 117-120.

[21] Duan Q, Chen P C, Zou Q H. Method for egg surface area estimation based on computer vision. Journal of Anhui Agricultural University, 2013; 40(2): 342 - 344. (in Chinese)

[22] Tu K, Pan L Q, Yang J L, Su Z P, Yu X. Dirt detection on brown eggs based on computer vision. Journal of Jiangsu University (Natural Science Edition), 2007; 28(3): 189-192. (in Chinese)

[23] United States Standards, Grades, and Weight Classes for Shell Eggs. AMS 56. 2000. Available: https://www.ams.usda.gov/ grades-standards/eggs. Accessed on [2016-5-12]. 